whereas pattern separation might subserve encoding ${ }^{10}$. Thus, it is interesting to note that a subsequent memory effect was located in the anterior hippocampus during $\mathrm{AB}$ study, a region previously associated with novelty and memory encoding ${ }^{11,12}$, whereas the subsequent memory effect for $\mathrm{AB}$ pairs during $\mathrm{AC}$ study was located in the posterior hippocampus and parahippocampal gyrus, regions that are considered to support retrieval ${ }^{11,12}$. In addition, the greatest subsequent memory effect for $\mathrm{AB}$ pairs during AC study in the between-subjects analysis was found in the medial prefrontal gyrus, an area previously associated with pattern completion and memory retrieval ${ }^{13}$. Although interesting, this pattern completion interpretation remains speculative. Pattern separation in the dentate gyrus is thought to precede and enable pattern completion in hippocampal CA1 region ${ }^{9}$. Thus, as the authors note, both may have occurred in this task; however, the spatial resolution of fMRI does not allow them to be separated.

In sum, Kuhl et al. ${ }^{1}$ provide us with a convincing demonstration that memories of old information can be reprocessed when new, similar information is learned and that this

\section{Speedy rod signaling}

reactivation is critical for the fate of the old memory. A central question that remains is whether the reactivation as detected reflects a restrengthening of memory through replay ${ }^{3}$ or an active trace change, as described for reconsolidation $^{4}$ and schema-dependent updating ${ }^{5}$. Replay occurs off-line and usually without perceptual input. When reactivation occurs in the presence of perceptual input, reactivated traces become unstable (a process that is critically dependent on the posterior hippocampus ${ }^{14}$ ) and require reconsolidation ${ }^{4}$. However, Kuhl et al. ${ }^{1}$ found the opposite: reactivation during novel learning protects memories from being forgotten, although on a much shorter time scale. A potential interpretation of these seemingly contradicting findings is that the reactivated memory and the current perceptual input can be integrated in such a way that the old memory is updated. Such integration has been shown to depend on the hippocampus and pre-existing knowledge ${ }^{5,15}$. Determining which of these processes underlie the findings of Kuhl et al. ${ }^{1}$ requires further investigation. Regardless, a neural correlate of memory reactivation has been very difficult to detect thus far, as the timing of reactivations is unknown.
Now, Kuhl et al. ${ }^{1}$ provide the neuroscience community with the opportunity to track reactivated memories and the ability to investigate the dynamic nature of memory.

\section{COMPETING INTERESTS STATEMENT}

The authors declare no competing financial interests.

1. Kuhl, B.A., Shah, A.T., DuBrow, S. \& Wagner, A.D. Nat. Neurosci. 13, 501-506 (2010).

2. McGaugh, J.L. Science 287, 248-251 (2000).

3. Sutherland, G.R. \& McNaughton, B. Curr. Opin. Neurobiol. 10, 180-186 (2000).

4. Nader, K. Trends Neurosci. 26, 65-72 (2003)

5. Tse, D. et al. Science 316, 76-82 (2007).

6. Anderson, M.C. J. Mem. Lang. 49, 415-445 (2003).

7. Underwood, B.J. Psychol. Rev. 64, 49-60 (1957).

8. Marr, D. Phil. Trans. R. Soc. Lond. B 262, 23-81 (1971).

9. Leutgeb, J.K., Leutgeb, S., Moser, M.-B. \& Moser, E.I. Science 315, 961-966 (2007)

10. Treves, A. \& Rolls, E.T. Hippocampus 4, 374-391 (1994).

11. Strange, B.A., Fletcher, P.C., Henson, R.N.A., Friston, K.J. \& Dolan, R.J. Proc. Natl. Acad. Sci. USA 96, 4034-4039 (1999).

12. Schacter, D.L. \& Wagner, A.D. Hippocampus 9, 7-24 (1999).

13. Jo, Y.S. et al. J. Neurosci. 27, 13567-13578 (2007).

14. Wang, S.-H., de Oliveira Alvares, L. \& Nader, K. Nat. Neurosci. 12, 905-912 (2009).

15. Kumaran, D., Summerfield, J.J., Hassabis, D. \& Maguire, E.A. Neuron 63, 889-901 (2009).
Rod photoreceptors in the mammalian retina allow vision under dim light conditions, when cones are not sufficiently activated. The rod light response, however, is relatively slow. Rods transmit their signals mainly to two effector cell types - to the ON bipolar cells by means of a synapse consisting of metabotropic glutamate receptors, and to adjacent cones by means of gap junctions. A third type of contact has been reported as well, between about $20 \%$ of rods in the mouse retina and a particular subset of OFF bipolar cells, the b2 cells. It was, however, not clear whether these contacts formed functional synaptic connections.

Now, Li, Chen and DeVries, on page 414 of this issue, characterize the electrophysiological properties of these contacts in slices from ground squirrel retina. The b2 bipolar cells, in contrast to the rod ON bipolar cells, express fast ionotropic AMPA-type glutamate receptors in their postsynaptic endings and could therefore mediate faster signaling.

The picture shows a b2 OFF bipolar cell (green) contacting a rod (red). The outer segment of the rod cell is stained blue for rhodopsin. Recording from such cell pairs, the authors found that kinetics of synaptic transmission between these cells is as fast and transient as transmission between cones and b2 bipolar cells and five to ten times faster than transmission between rods and rod ON bipolar cells or rods and cones. In other respects, too, such as synaptic vesicle replenishment, the rod-b2 OFF bipolar cell synapses resemble cone-b2 OFF bipolar cell synapses.

Although this study does not tease out any specific contribution of the new fast transmission circuit component to rod-mediated vision, the discovery of fast rod signaling is surprising in itself. One may speculate that at intermediate light intensities, cones and a subset of rods collaboratively activate the b2 celldriven OFF circuitry. We look forward to future work revealing the physiological significance of this new input.

Annette Markus

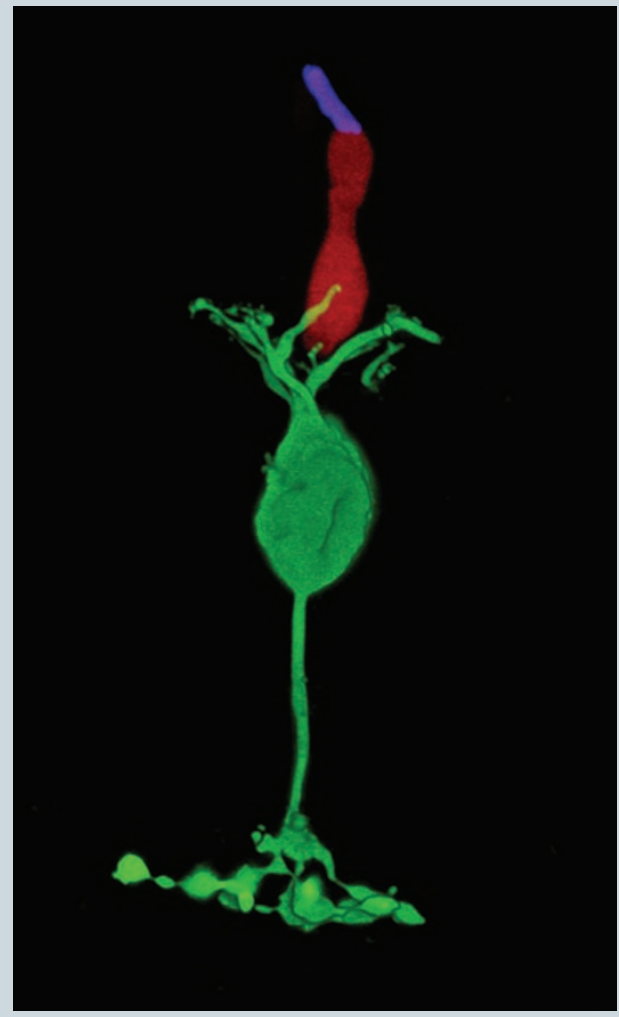

